\title{
The Root Lesion Nematode Effector Ppen10370 Is Essential for Parasitism of Pratylenchus penetrans
}

\author{
Paulo Vieira, ${ }^{1,2}$ Cláudia S. L. Vicente, ${ }^{3,4}$ Jordana Branco, ${ }^{3}$ Gary Buchan, ${ }^{5}$ Manuel Mota, ${ }^{3}$ and \\ Lev G. Nemchinov ${ }^{1, \dagger}$ \\ ${ }^{1}$ United States Department of Agriculture-Agricultural Research Service (USDA-ARS), Molecular Plant Pathology Laboratory, \\ Beltsville, MD 20705-2350, U.S.A. \\ ${ }^{2}$ School of Plant and Environmental Science, Virginia Tech, Blacksburg, VA 24061, U.S.A. \\ ${ }^{3}$ NemaLab, MED-Mediterranean Institute for Agriculture, Environment and Development, Departamento de Biologia, Escola de \\ Ciências e Tecnologia, Universidade de Évora, Pólo da Mitra, Ap. 94, 7006-554 Évora, Portugal \\ ${ }^{4}$ INIAV, I.P.-Instituto Nacional de Investigação Agrária e Veterinária, Quinta do Marquês, 2780-159 Oeiras, Portugal \\ ${ }^{5}$ Electron \& Confocal Microscopy Unit, USDA-ARS, Beltsville, MD 20705, U.S.A.
}

Accepted 5 January 2021.

The root lesion nematode Pratylenchus penetrans is a migratory species that attacks a broad range of crops. Like other plant pathogens, $P$. penetrans deploys a battery of secreted protein effectors to manipulate plant hosts and induce disease. Although several candidate effectors of $P$. penetrans have been identified, detailed mechanisms of their functions and particularly their host targets remain largely unexplored. In this study, a repertoire of candidate genes encoding pioneer effectors of $P$. penetrans was amplified from mixed life stages of the nematode, and candidate effectors were cloned and subjected to transient expression in a heterologous host, $\mathrm{Ni}$ cotiana benthamiana, using potato virus $\mathrm{X}$-based gene vector. Among seven analyzed genes, the candidate effector designated as Ppen10370 triggered pleiotropic phenotypes substantially different from those produced by wild type infection. Transcriptome analysis of plants expressing Ppen10370 demonstrated that observed phenotypic changes were likely related to disruption of core biological processes in the plant due to effector-originated activities. Cross-species comparative analysis of Ppen10370 identified homolog gene sequences in five other Pratylenchus species, and their transcripts were found to be localized specifically in the nematode esophageal glands by in situ hybridization. RNA silencing of the Ppen10370 resulted in a significant reduction of nematode reproduction and

This paper is dedicated to the memory of Gary Buchan, who passed away on January 12, 2021 due to complications from COVID-19.

${ }^{\dagger}$ Corresponding author: L. G. Nemchinov; lev.nemchinov@usda.gov

Funding: This project was supported by the United States Department of Agriculture Agricultural Research Service CRIS project number 8042 21000-300-00D, by the National Funds through Fundação para a Ciência e a Tecnologia (FCT, Foundation for Science and Technology) under the projects UIDB/05183/2020 and PTDC/AGR-PRO/2589/2014 (to M. Mota and J. Branco) and by a FCT postdoctoral fellowship SFRH/BPD/116030/2016 (to C. S. L. Vicente).

*The $e$-Xtra logo stands for "electronic extra" and indicates there are supplementary figures and supplementary tables published online.

The author(s) declare no conflict of interest.

Copyright (C) 2021 The Author(s). This is an open access article distributed under the CC BY-NC-ND 4.0 International license. development, demonstrating an important role of the esophageal gland effector for parasitism.

Keywords: nematode-plant interactions, nematode-induced plant genes, nematode effectors, transient expression using potato virus $\mathrm{X}(\mathrm{PVX})$-based vector

Root lesion nematodes (RLN) (Pratylenchus spp.) are economically important pathogens that inflict significant damage and yield losses on a wide range of crops (Castillo and Vovlas 2007). Pratylenchus penetrans is one of the most important nematodes of the genus attacking nearly 400 species including high-value crops, grasses, and fruit trees (Castillo and Vovlas 2007). All stages of $P$. penetrans, except eggs and first-stage juveniles, are motile (Zunke 1990), and several generations of nematodes can develop during the life span of the crop. Pratylenchus spp. are migratory endoparasitic nematodes and their mobility throughout their life cycle causes massive damage to the root system, predisposing the roots to secondary infections by other soil-borne pathogens (Castillo and Vovlas 2007). As RLN migrate into the root of the host plant, they feed mainly from the cortex cells to gain access to the nutrients. Due to their different parasitic strategies, management approaches for controlling RLN are often ineffective and mostly rely on sanitation and the use of nematicides (Davis and MacGuidwin 2000).

Plant-parasitic nematode (PPN)-host interactions are mediated by a complex molecular dialogue, which involves the nematode secretion of different sets of proteins, called effectors (Vieira and Gleason 2019). These effectors are critical components of parasitism and are involved in all stages of nematode-host interactions. Like other PPN, P. penetrans effector genes are mainly expressed in the esophageal glands of the nematode and the corresponding proteins are ultimately secreted into the plant through a hollow stylet to manipulate host cell physiology and to promote infection (Vieira and Gleason 2019). Given the importance of RLN effectors in parasitism, their functional roles and mechanism of action are of particular interest for understating the molecular pathways of nematode-host interactions as well as for development of nematode control strategies.

As a first step toward elucidating the molecular basis of $P$. penetrans parasitism strategy, we have used bioinformatic analysis to identify a set of candidate effectors in the transcriptome of $P$. penetrans. Based on the presence of putative signal peptides, absence of transmembrane domains, and 
expression patterns during plant infection, we generated a catalog of predicted secreted proteins for this species (Vieira et al. 2015). Data mining of a transcriptome library originated specifically from the esophageal glands, coupled with in situ hybridization assays, validated a set of 23 candidate effector genes for P. penetrans (Vieira et al. 2018; Vicente et al. 2019). These encompassed a suite of genes encoding cell walldegrading enzymes (CWDEs) and a pectin methylesterase gene, which had not been found in any nematode species prior to this study (Vicente et al. 2019). Nematode CWDEs are often implicated in the softening and degradation of the plant cell wall, allowing nematode penetration and migration within the host tissues (Smant et al. 1998). A few other genes included those frequently recognized as part of the nematode-host secretome, for instance fatty acid- and retinol-binding proteins, venom allergen-like proteins and an array of diverse classes of putatively secreted proteases involved in protection against host defenses (Vieira et al. 2018).

A prominent feature of this comparative analysis was the presence of a significant proportion of $P$. penetrans transcripts encoding putative proteins without any known function or annotation (Vieira et al. 2015). From this list of putative secreted proteins, a set of seven genes was confirmed to be expressed within the esophageal glands of $P$. penetrans (Vieira et al. 2018). The molecular mechanisms by which these effector-like proteins contribute to the parasitic behavior of the nematode and their host targets are still largely unknown (Vieira et al. 2015, 2018). Therefore, exploring the roles of these pioneer effectors of $P$. penetrans could provide an important view on the molecular mechanisms underlying RLN parasitism-a knowledge that can be useful for the development of new control measures against this resilient group of nematodes.

In this study, we cloned, sequenced, and characterized by transient expression methodology a set of seven pioneer candidate effectors of $P$. penetrans. The methodology relied on using potato virus X (PVX)-based vector, a well-established tool for high-throughput functional screening and rapid in-planta studies (Lacomme and Chapman 2008). Our main focus was to advance our knowledge on the functional roles of these pioneer effectors during parasitism of P. penetrans. As a result of this work, one of the core effector genes of $P$. penetrans and other Pratylenchus spp., designated as Ppen10370, was identified. Ectopic expression of Ppen10370 in planta induced a pronounced stress response in the host, which included upregulation of a significant number of genes essential for both plant defense and for the prospective functions of the effector during parasitism. RNAinduced silencing of this gene resulted in a decreased number of nematodes recovered from infected carrot roots, suggesting its important role in the nematode parasitism.

\section{RESULTS}

\section{Selection of Pratylenchus penetrans candidate effector genes and gene structure.}

Our previous studies identified and validated 23 candidate effectors from $P$. penetrans, whose transcripts were specifically localized within the nematode esophageal glands (Vieira et al. 2018; Vicente et al. 2019). From this list, seven candidates were considered pioneer genes without any annotation or predictive function. These novel genes were selected for further analysis to expand their characterization and to reveal their functional roles in parasitism. The full-length genomic and cDNA sequences of each individual effector were cloned and the overall gene features were determined (Table 1). All genomic sequences revealed the presence of introns (1 to 3), with the exception of Ppen11402 (Supplementary Fig. S1). Sequence analysis showed that the full-length cDNA clones encoded proteins with a predicted signal peptide consisting of 15 to 26 amino acid residues (Table 1), which is considered a key feature for secretion. This set of cDNAs encode for relatively small proteins, ranging from 74 to 176 amino acids (7.91 to $11.44 \mathrm{kDa}$ ). Noteworthy, five genes in this set display a high percentage of proline residues, which can represent up to $36.5 \%$ of the mature protein (Table 1).

\section{Identification of homologs of candidate effector genes.}

To identify potential homologs among other PPN (including different RLN species), each sequence was screened against publicly available transcriptomic and genomic datasets using BLAST analysis. Reciprocal BLASTp and tBLASTn searches allowed the identification of transcripts/genes with high identity against transcriptomes of other RLN, confirming the presence of potential homologs of at least four $P$. penetrans effector genes (Fig. 1A). No significant BLAST hits were obtained against nematodes of different genera or other organisms, suggesting that these effectors are specific to RLN.

Remarkably, significant BLAST hits against Ppen10370 were found in all Pratylenchus species tested (i.e., P. brachyurus, P. coffeae, P. thornei, P. neglectus, and P. zeae). Ppen10370 is part of a small family of related genes (about three sequences) in $P$. penetrans, with all of them coding for putative secreted proteins. With the exception of $P$. zeae, from which a partial transcript was recovered, all predicted proteins of the remaining RLN species possess a predicted signal peptide needed for secretion (Fig. 1B). On the basis of sequence homology analysis, the conserved amino acids between the predicted proteins among other RLN were mostly distributed at the C-terminal region, preceded by a putative predicted linker (Fig. 1B). PCR amplifications of the full-length coding sequence (CDS) of $P$. neglectus and $P$. thornei confirmed our in silico analysis. Since localization of transcripts in the secretory esophageal glands is often used to validate candidate effector genes with a potential role in parasitism, we investigated the localization of $P$. neglectus and $P$. thornei homologs of Ppen 10370 by in situ hybridization. Consistent with the results obtained for Ppen 10370 of $P$. penetrans, the localization of transcripts was specific to the secretory esophageal glands of $P$. neglectus and $P$. thornei (Fig. 1C). Taken together, the specific transcript localization within the esophageal glands and protein sequence similarity suggest that these effectors may share one or more similar functions in different RLN species.

\section{Gene expression patterns of $P$. penetrans effectors during infection of different host plants.}

To validate the expression profile of the seven pioneer genes during plant infection, transcript abundance was evaluated using available transcriptomic libraries of different plants infected with $P$. penetrans (Vieira et al. 2015; 2019). The analysis confirmed active transcription of this set of genes during nematode infection of alfalfa and soybean plants (Fig. 2A). To substantiate the in silico analysis, semiquantitative reverse transcription PCR (RT-PCR) assays were performed with independent cDNA libraries derived from nematode-infected alfalfa cv. Baker at 3 and 7 days after infection (DAI). Similar gene expression patterns were obtained for the same set of genes (Fig. 2B). These data illustrated that the temporal expression of these genes correlates with $P$. penetrans infection in roots of different host plants.

\section{Induction of different phenotypes in planta during ectopic expression of $\boldsymbol{P}$. penetrans candidate effectors.}

To further dissect potential functions of the seven pioneer genes, we tested their ability to elicit phenotypic responses in the model plant Nicotiana benthamiana via transient expression 
assay using a PVX-based vector. Each effector sequence encoding the mature protein (without the signal peptide) was amplified and cloned into the PVX-based vector (pP2C2S) (Chapman et al. 1992). For each assay, a minimum of three independent plants were tested and the PVX wild-type (WT, no insert) construct was used as a control. The severity and type of symptoms induced by the effectors varied (Fig. 3; Supplementary Fig. S2). The most pronounced phenotype occurred in plants inoculated with the recombinant virus expressing the Ppen10370 effector gene, whose homologs were found in all Pratylenchus species described above. The inoculated plants were systemically infected and displayed evident vein necrosis

Table 1. Molecular characterization of the seven pioneer candidate genes of Pratylenchus penetrans, including their genomic and coding region features ${ }^{\mathrm{a}}$

\begin{tabular}{|c|c|c|c|c|c|c|c|c|c|c|}
\hline $\begin{array}{l}\text { Candidate } \\
\text { effector }\end{array}$ & $\begin{array}{c}\text { Transcript } \\
\text { length }\end{array}$ & $\begin{array}{c}5^{\prime} \\
\text { UTR }\end{array}$ & $\begin{array}{c}\text { Coding sequence } \\
\text { (nt) }\end{array}$ & $\begin{array}{c}\mathbf{3}^{\prime} \\
\text { UTR }\end{array}$ & $\begin{array}{c}\text { Poly- } \\
\text { A }\end{array}$ & $\begin{array}{c}\text { Genome } \\
\text { (nt) }\end{array}$ & $\begin{array}{l}\text { Protein } \\
\text { (aa) }\end{array}$ & PI & $\begin{array}{c}\text { MW } \\
\text { (kDa) }\end{array}$ & $\begin{array}{c}\text { Proline content }{ }^{\mathbf{b}} \\
(\%)\end{array}$ \\
\hline Ppen11402 & 456 & 26 & 240 & 177 & Yes & 240 & 79 & 10.58 & 8.79 & 7.8 \\
\hline Ppen8004 & 484 & 43 & 279 & 143 & Yes & 488 & 92 & 3.41 & 9.28 & 31 \\
\hline Ppen7984 & 405 & 13 & 225 & 156 & Yes & 303 & 74 & 11.44 & 7.91 & 36.5 \\
\hline Ppen16605 & 615 & 76 & 309 & 217 & Yes & 702 & 102 & 4.18 & 10.67 & 27.1 \\
\hline Ppen12016 & 663 & 52 & 390 & 205 & Yes & 476 & 129 & 9.86 & 12.97 & 24.8 \\
\hline Ppen10370 & 601 & 84 & 306 & 190 & Yes & 363 & 101 & 9.33 & 10.93 & 18.7 \\
\hline Ppen11230 & 746 & 6 & 531 & 209 & Yes & 747 & 176 & 10.94 & 19.63 & 8.6 \\
\hline
\end{tabular}

${ }^{a}$ Genes are sorted based on the size of their respective predicted encoded proteins. UTR $=$ untranslated region, PI $=$ isoelectric point, and MW $=$ molecular weight.

${ }^{\mathrm{b}}$ In mature protein.
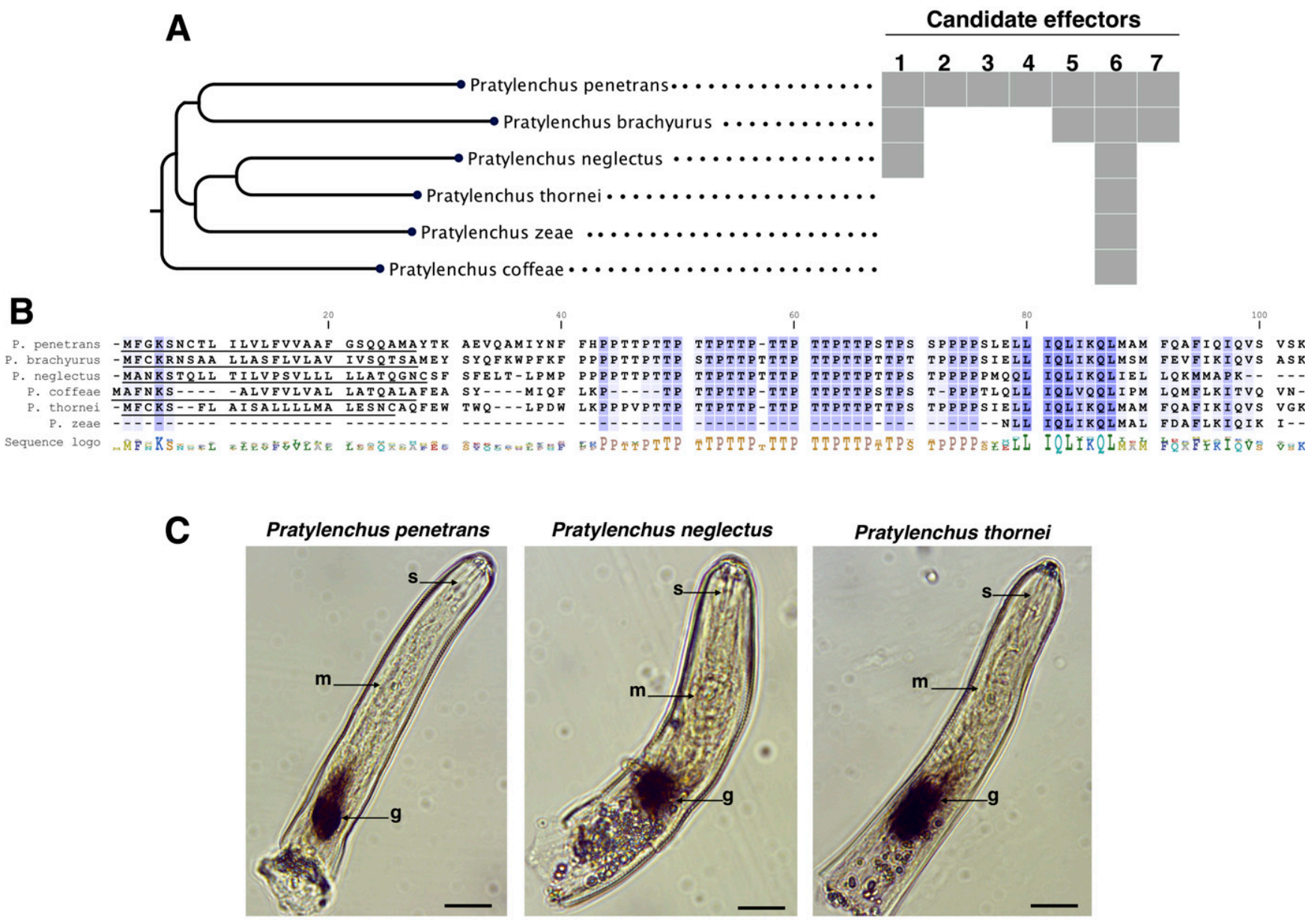

Fig. 1. Identification of homologs of candidate effector genes of Pratylenchus penetrans in other root lesion nematodes species. A, BLAST analysis against transcriptome datasets of other Pratylenchus species using P. penetrans effector candidates as query. The schematic phylogeny is based on the D2D3 region of the 28S rRNA gene: 1, Ppen11402; 2, Ppen8004; 3, Ppen7984; 4, Ppen16605; 5, Ppen12016; 6, Ppen10370; 7, Ppen11230. B, Amino acid sequence alignment of the Ppen 10370 effector protein from P. penetrans and its putative homologs from other Pratylenchus species. The full-length predicted proteins were obtained from cloned coding sequences of $P$. neglectus and $P$. thornei or were predicted from the transcriptome assembly of $P$. brachyurus and $P$. coffeae. For $P$. zeae only a partial transcript was obtained. Predicted signal peptides are underlined. Conserved residues among species are indicated by dark blue shading, whereas similar residues are represented in light blue using a threshold for shading of $60 \%$ similarity. C, Detection of gene transcripts of $P$. penetrans Ppen 10370 and putative homologs of the gene from P. neglectus and P. thornei by in situ hybridization. Transcripts were detected in the nematode esophageal glands, using the corresponding antisense digoxigenin-labeled cDNA probes. $\mathrm{g}=$ esophageal gland, $\mathrm{m}=$ median bulb, and $\mathrm{s}=\mathrm{stylet}$. Scale bars $=20 \mu \mathrm{m}$. 
on the leaves (Fig. 3A and B) and lesion-like areas on the petiole and stem of $N$. benthamiana (Fig. 3C). The plants appeared smaller in comparison with the ones infected with the empty PVX/WT vector. Expression of the Ppen10370 gene was confirmed by RT-PCR with the effector-specific primers (Supplementary Fig. S3). Two other constructs (PVX/Ppen8004 and PVX/Ppen16605) induced small lesion-like symptoms on the leaves (Fig. 3D through I). Plants expressing the remaining genes displayed typical mosaic symptoms with dark light-green patches (Supplementary Fig. S2), similar to the phenotype induced by the PVX/WT (Fig. 3J through L). In planta expression of these nematode effectors was also confirmed by RT-PCR (Supplementary Fig. S4).

Considering that the most acute phenotypes were a result of PVX/Ppen 10370 infection (Fig. 3A and B), expression assays with this construct were also performed in N. tabacum and tomato plants (Lycopersicon esculentum). In tomato plants, spot-like necrotic areas could be observed in systemically infected leaves expressing PVX/Ppen10370 (Supplementary Fig. S5A), while, in tobacco, systemically infected leaves displayed necrotic lesions (Supplementary Fig. S5B). Plants of both species inoculated with PVX/WT had typical PVX mosaic-like symptoms (Supplementary Fig. S5).

\section{Subcellular localization of Ppen10370}

in $N$. benthamiana leaves.

To gain insight into the subcellular localization of Ppen10370, the coding sequence of the gene without signal peptide was fused to the enhanced green fluorescent protein (Ppen10370 $\Delta$ SP: eGFP) in the binary vector pK7FWG2.0 (Karimi et al. 2002) and transiently expressed in $N$. benthamiana, using agroinfiltration. Western blot assays confirmed the presence of the Ppen10370 $\Delta$ SP: eGFP fusion protein in the agroinoculated leaves of $N$. benthamiana (Supplementary Fig. S6). The fusion protein was found to be localized in the endoplasmic reticulum (ER) network and surrounding the nucleus (Fig. 4A and B). Fluorescence of free eGFP used as a control was observed throughout the cytoplasm and the nucleus of $N$. benthamiana cells (Fig. 4C).
Differential gene expression analysis in plants transiently expressing PVX/Ppen10370.

To decipher a molecular basis of the Ppen10370-induced phenotypic abnormalities, we analyzed transcriptome responses in $N$. benthamiana plants infected with the recombinant PVX/Ppen 10370 vector. For the RNA-sequencing (RNA-seq) analysis, noninoculated, systemically infected leaves showing symptoms described above were selected (Fig. 3A and B; Supplementary Fig. S3). Plants inoculated with PVX/WT were used as a control.

A total of around 268 million clean reads were generated from six RNA-seq libraries (three PVX/Ppen10370 and three PVX/WT [Supplementary Table S1]). The reads were mapped to the reference genome of PVX (GenBank number M72416.1) and to a publicly available genome of $N$. benthamiana. The average percentage of the total number of reads mapped to the corresponding genomes is shown in Supplementary Table S1. Differentially expressed genes (DEGs) unique to the PVX/Ppen10370infected plants were depicted against the background of a wildtype PVX infection. In total, 1,086 DEGs with broad biological roles were identified in the PVX/Ppen10370-infected plants (Supplementary Table S2). Of this group, 721 DEGs were significantly upregulated (fold change $>2$ and false discovery rate $[\mathrm{FDR}]<0.05$ ) and 365 DEGs were downregulated (Fig. $5 \mathrm{~A})$. A twofold higher prevalence of the upregulated genes indicated that the overall impact of Ppen10370 lies in the activation of the host biological and cellular processes thus leading to the phenotypic changes observed in the infected plants.

\section{Functional analyses of DEGs.}

Upregulated DEGs. Gene ontology (GO) enrichment analysis of the upregulated DEG resulted in several overrepresented categories, including the most highly represented term 'response to stress' (GO:0006950) in the biological process class (Fig. 5B; Supplementary Table S3A). Several GO terms in the molecular function (MF) category were also significantly enriched (false discovery rate $[\mathrm{FDR}]<0.05)$, namely, "transferase activity" (GO:
A

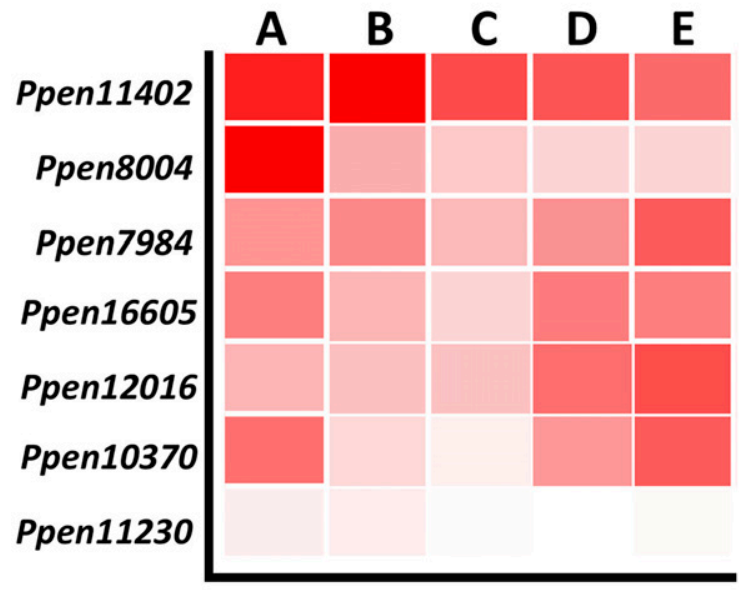

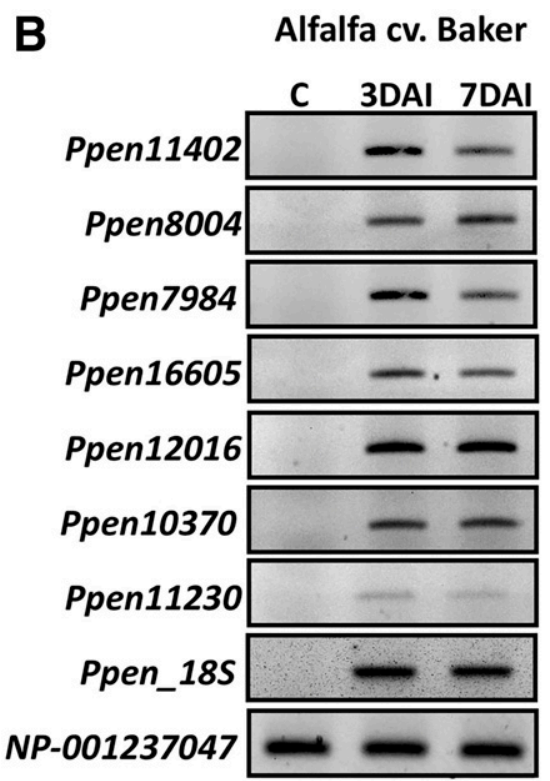

Fig. 2. Gene expression levels of Pratylenchus penetrans candidate effectors in planta. A, Expression levels were calculated using reads per kilobase of transcript per million mapped reads (FPKM) values for each effector gene, using public mRNA-seq datasets originating from A, nematodes only, $\mathbf{B}$ and $\mathbf{C}$, soybean plants infected with P. penetrans at 3 and 7 days after infection (DAI), respectively (BioProject ID PRJNA304159), and D and E, two alfalfa cultivars, cv. Baker and MNGNR-16, respectively, infected with $P$. penetrans at 7 DAI (BioProject ID PRJNA547347). B, Semiquantitative reverse transcription PCR validating the expression levels of the different nematode effector genes from cDNA libraries derived from nematode-infected alfalfa roots (cv. Baker) collected at 3 and 7 DAI. 
0016758), "endopeptidase activity" (GO:0004866), and "antioxidant activity" (GO:001 6209). In the cellular component class, apoplast (GO:0048046) was the only enriched GO term (Fig. 5C). To further delineate the mechanisms behind PVX/Ppen10370induced phenotypes, we performed a manual sorting of those DEGs whose involvement may shed light on the functional activity of this particular nematode effector gene. Two most-relevant groups provisionally involved in i) host defense responses to the effector and ii) effector-originated functions and activities were apparent (Table 2). While the defense-related cluster included upregulated genes encoding proteins with well-known roles in stress response, such as NAC and WRKY transcription factors, pathogenesis-related (PR) proteins, chitinases, chloroplast proteins (Table 2), it also contained genes encoding proteins participating in detoxification, toxin removal and attenuation of oxidative stress, such as detoxification efflux carrier genes. The second group of upregulated genes presumably related to the functioning of the effector protein included 25 heat shock proteins (HSPs) and 15 ubiquitin-related proteins (URPs) exclusively upregulated in the Ppen10370-expressing plants. Kyoto Encyclopedia of Genes and Genomes (KEGG) pathway enrichment analysis reflected these observations indicating the importance of "protein processing in endoplasmic reticulum", "proteasome", and "antioxidant and detoxification-related" activities in host responses to the nematode effector (Fig. 6A; Supplementary Table S4A).

Downregulated DEGs. GO enrichment analysis of the downregulated DEGs emphasized the importance of several metabolic processes associated with the host reaction to this nematode

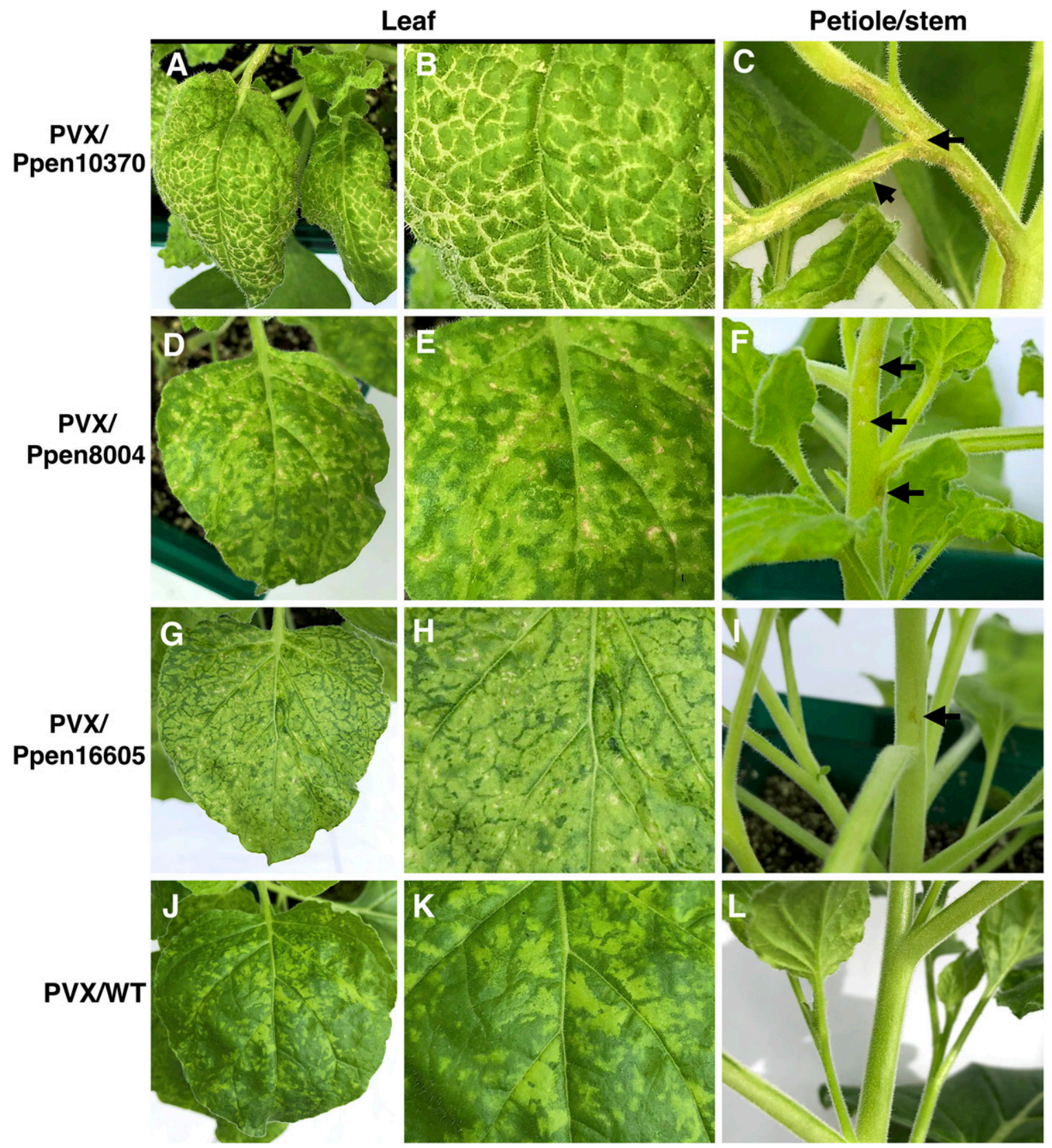

Fig. 3. Phenotypic changes in Nicotiana benthamiana plants induced by the infection with recombinant potato virus X (PVX) carrying genes encoding different candidate effectors of Pratylenchus penetrans. A to C, Expression of Ppen 10370 gene induced vein necrosis on leaves and necrosis on the petiole and stem of the infected plants (indicated by arrows). D to F, Expression of Ppen 8004 gene induced necrotic spots on leaves and stem of the infected plants (indicated by arrows). G to I, Expression of Ppen16605 gene induced chlorosis and small-necrotic spots on leaves and lesion spots on the stem of the infected plants (indicated by arrows). $\mathbf{J}$ to $\mathbf{L}$, Typical mosaic-like symptoms induced by PVX-based vector without insert (PVX/WT) on leaves of $N$. benthamiana plants. No lesion-like symptoms were observed. All photos were taken 14 days after inoculation. 
effector gene, particularly genes in the MF domain associated with "glucosyltransferase activity" (GO:0035251) and "sucrose synthase activity" (GO:0016157) (Fig. 5B; Supplementary Table S3B). Individual sorting of the DEGs that could be redundant or unnoticed during GO and KEGG analyses resulted in the following groups of DEGs that are likely to be involved in response to the effector (Table 3). A large number of downregulated photosynthesis-related genes is consistent with the general effect of the virus infection on the host plants (Zhao et al. 2016), which appeared to be further enhanced by the expression of Ppen10370, leading to leaf chlorosis and extensive veinal necrosis. Other promising downregulated DEGs included several $P R$ genes, such as $P R I$ (Niben101Scf23606g00005.1) and PR5 (Niben101Scf08044g00001.1), and leucine-rich repeat (LRR) receptor-like serine/threonine kinase genes. The outcome of KEGG analysis was more generalized, resulting in a large group of genes involved in main metabolic pathways (Fig. 6B; Supplementary Table S4B). DEGs involved in terpenoid backbone biosynthesis were also overrepresented in KEGG pathways. Terpenoids are important secondary metabolites with diverged functions in plant growth, development, and protection against environment (Pichersky and Raguso 2018).

\section{Quantitative real-time PCR (qPCR) confirmation of the transcriptomic data.}

Expression patterns identified by computational analysis of RNA-seq-generated data were further confirmed by qPCR assays with RNA extracted from PVX/Ppen 10370 plants. A set of 25 genes was selected from the pathways most affected by the expression of the Ppen10370 (Table 4). Overall, the results obtained by qPCR and RNA-seq were consistent and followed similar expression patterns.

\section{Silencing of the Ppen10370 gene hampered nematode development.}

Based on the same reasoning that transient expression of Ppen10370 led to the pronounced phenotypes in N. benthamiana, thus indicating its important role in the parasitism, we conducted a gene-silencing assay to directly assess its potential contribution to host parasitism. It was accomplished by soaking mixed nematode stages (juveniles and adults) in a solution containing a 212-bp double-stranded RNA (dsRNA) fragment targeting the Ppen10370 gene. As control treatments, nematodes were soaked in a solution with a GFP-derived dsRNA fragment or in a buffer solution only.
qPCR analyses showed a significant reduction of the relative transcript abundance (approximately $60 \%, P<0.05$ ) in nematodes silenced with the Ppen10370 dsRNA fragment as compared with the expression levels in both control treatments (Fig. 7A). Soaking nematodes in dsRNA solution targeting the Ppen10370 gene did not result in any changes in their movement or survival rates, as observed after $24 \mathrm{~h}$ of soaking. When Ppen10370 dsRNA-treated nematodes were placed on carrot disks and allowed to develop for a period of 6 weeks, a significantly reduced number of nematodes $(P<0.05)$ was recovered from the carrot disks compared with the number of nematodes recovered from the control treatments (Fig. 7B), suggesting that this gene is required for successful establishment of parasitism.

\section{DISCUSSION}

In this work, we cloned the full-length protein-coding sequences of seven pioneer effectors based on their potential involvement in parasitism. An in-depth investigation into the biological functions of Ppen10370, which, based on the results of this work, were assumed to play important roles in parasitism, was performed by transient expression in a model host, utilizing phenotypical evaluation, high-throughput sequencing, differential gene expression analysis, and dsRNA silencing. As a result, potential biological roles of this specific candidate effector in parasitism were proposed and molecular mechanisms of their impact on host plants were revealed.

Recently, we applied a PVX-based transient expression approach for the characterization of different CWDEs that function as effector proteins of P. penetrans (Vicente et al. 2019; Vieira and Nemchinov 2020). As a result of that investigation, the biological roles of an expansin-like effector in nematode parasitism were proposed and the putative cellular targets of the protein were identified (Vieira and Nemchinov 2020). In continuation of this work, we have further explored this methodology toward deciphering functions of pioneer effector genes (i.e., without a putative function or annotation) of $P$. penetrans, which presently remain poorly understood. Of the seven candidate effectors screened in this work, expression of three effectors resulted in the induction of distinct phenotypes not observed in plants infected with PVX/WT. Based on the phenotypic observations, we proceeded to an in-depth characterization of the Ppen10370 gene, which presented the most distinct phenotypes when ectopically expressed in planta.
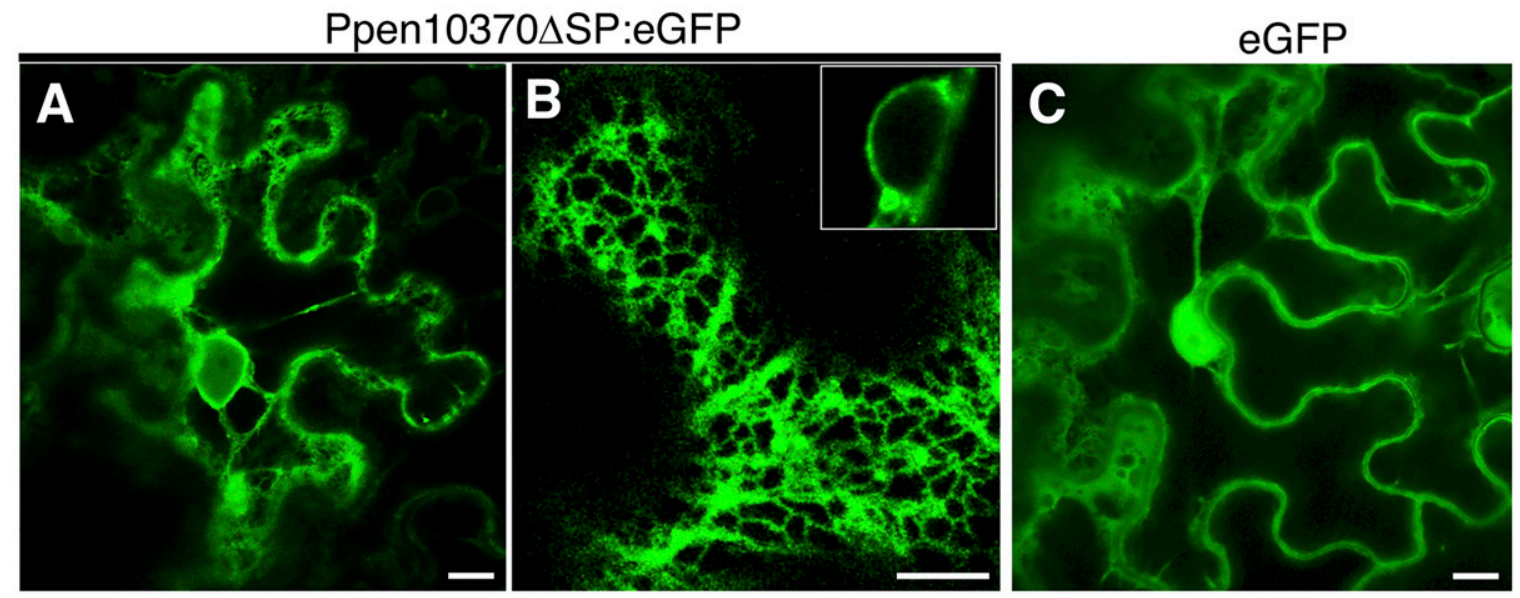

Fig. 4. Subcellular localization of Ppen 10370 in Nicotiana benthamiana leaves. A and B, Fluorescent signal produced by the fusion Ppen10370 $\Delta$ SP:eGFP protein and detected in the endoplasmic reticulum (ER) network and surrounding the nuclear membrane of $N$. benthamiana leaf cells. The insert in B shows ER around the nucleus. C, Localization of the free enhanced green fluorescent protein (eGFP) in the cytoplasm and nucleus of $N$. benthamiana leaves. For confocal laser scanning microscopy, leaf samples were taken $48 \mathrm{~h}$ postinoculation. Scale bar $=5 \mu \mathrm{m}$. 
With the exception of RLN, the predicted protein encoded by the Ppen 10370 gene did not show any similarity to other proteins deposited in the publicly available datasets at the time of this investigation. In silico analysis of this particular gene resulted in the identification of additional homolog sequences in the available transcriptome and genomic data sets for five other RLN species. The occurrence of Ppen10370 homologs in several species of the genus Pratylenchus, expression and localization of the gene transcripts within their secretory esophageal glands, and gene silencing results supported the idea that this gene might be a core feature related to the parasitic mode of life in this group of nematodes.

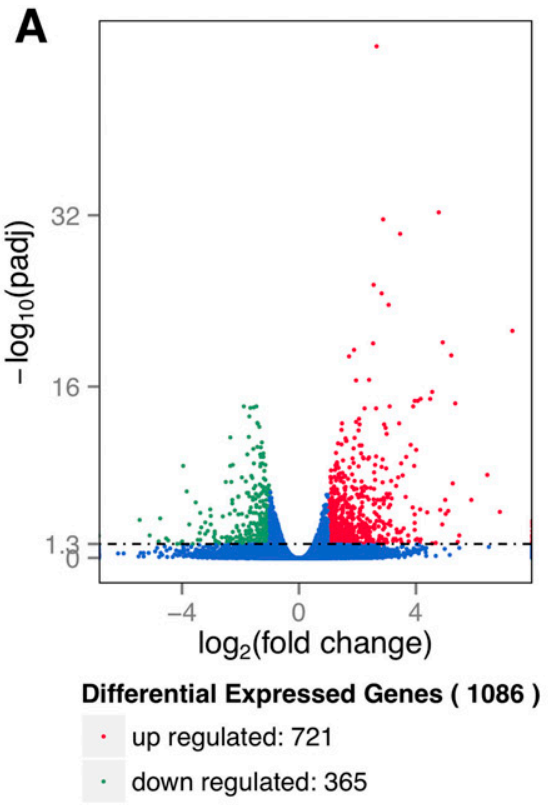

B

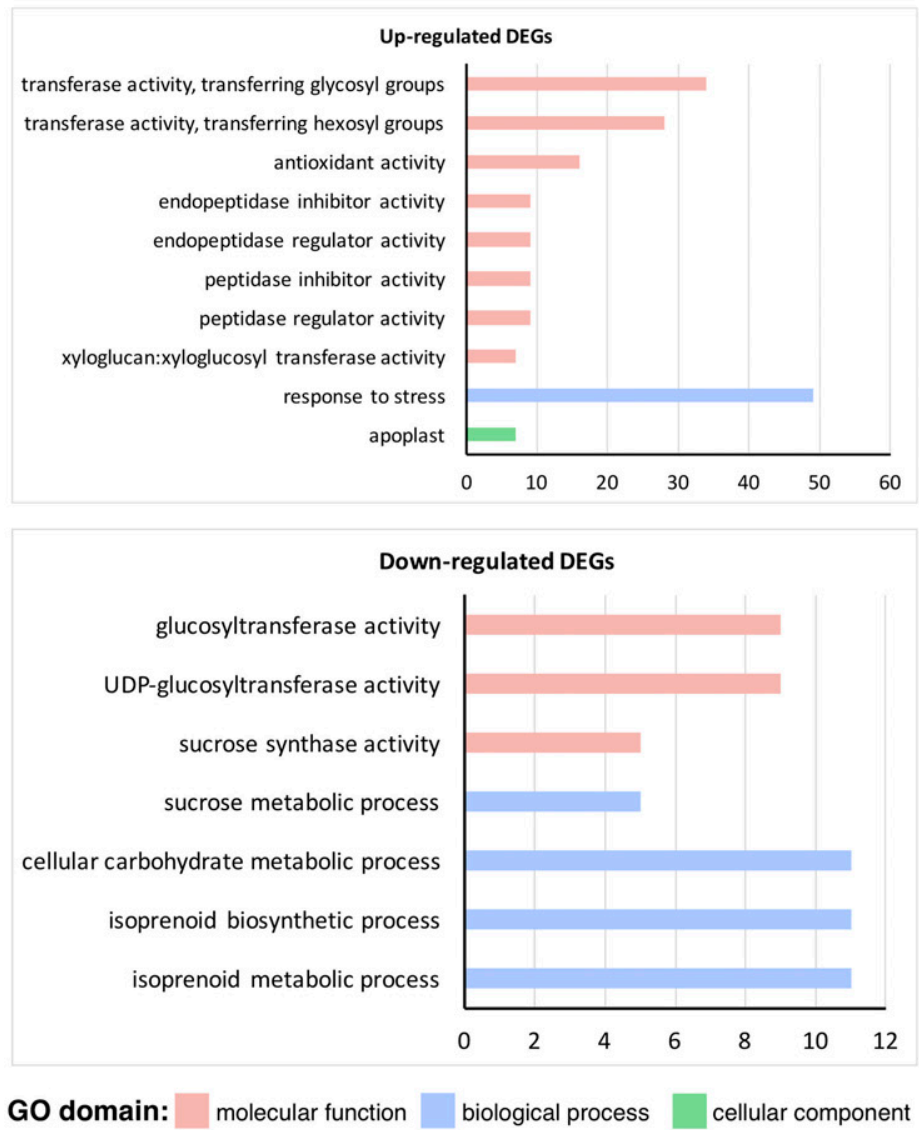

Fig. 5. Gene expression profiling of Nicotiana benthamiana plants inoculated with RNA transcripts derived from the PVX/Ppen10370 recombinant vector and from the PVX-based vector without insert (PVX/WT). A, Volcano plot representation shows the total number of differentially expressed genes (DEGs) significant between PVX/Ppen10370 and PVX/WT. B, Functional gene ontology (GO) enrichment analysis of DEG sets identified between plants inoculated with PVX/Ppen10370 and PVX/WT. The enriched GO terms (false discovery rate <0.05) are plotted in relation to the number of DEGs. 
Transient expression of Ppen10370 in N. benthamiana plants followed by high-throughput sequencing and analysis of gene expression changes offered unique insights into the biological activity of this protein and suggested that Ppen10370 could have toxic activities similar to those associated, for example, with Nep 1-like (necrosis and ethylene-inducing peptide1) proteins produced by many bacterial, fungal, and oomycete species (Ottmann et al. 2009). Interestingly, the Nep1-like protein, which is presumably involved in the interaction with Nep 1 as well as in a plant defense signaling pathway, was upregulated more than twofold in the PVX/Ppen 10370 plants (Supplementary Table S3).

The second group of upregulated genes related to the ectopic expression of Ppen10370 included HSPs and URPs. It is noteworthy, that these genes were previously found to be upregulated during transient expression of another candidate effector from
P. penetrans, Pp-EXPB1 (Vieira and Nemchinov 2020). HSPs are responsible for protein folding, assembly, translocation, and degradation under stress and normal conditions (Park and Seo 2015). Twenty-five HSPs solely upregulated in Ppen10370-expressing plants suggested that a large-scale protein maintenance and stabilizing activity was induced by the expression of this nematode effector in plants. Although no supporting evidence could presently be found in the literature, hypothetically, the effector could initiate the same activity during $P$. penetrans parasitism to promote assembly and sustainment of the nematode-secreted proteins. Ubiquitin proteins play important roles in plant biology, not only related to protein modification and degradation systems but also to plant hormone signaling pathways (Bartel and Citovsky 2012). The ubiquitin-protein ligases that were found to be activated by Ppen 10370 in this study function as auxin and jasmonate receptors (Bartel and Citovsky 2012). Recently, the

Table 2. Selected proteins encoded by genes upregulated in response to transient expression of the Ppen10370 effector gene of Pratylenchus penetrans in Nicotiana benthamiana plants

\begin{tabular}{|c|c|c|}
\hline Name & Relevant functional roles & No. of genes \\
\hline Heat shock proteins & Protein assembly and degradation (Park and Seo 2015) & 25 \\
\hline Chloroplastic proteins & Photosynthesis; defense signaling (Nomura et al. 2012; Serrano et al. 2016) & 22 \\
\hline NAC transcription factors & Plant defense (Nuruzzaman et al. 2013; Pandey and Somssich 2009) & 15 \\
\hline Glutathione S-transferases & Detoxification; attenuation of oxidative stress (Gullner et al. 2018) & 13 \\
\hline Ubiquitin-related proteins & Protein regulation and degradation; immune signaling (Varshavsky 2008; Marino et al. 2012) & 12 \\
\hline WRKY transcription factors & Stress response (Pandey and Somssich 2009) & 7 \\
\hline Receptor-like serine threonine kinases & Signal perception; pathogen recognition (Afzal et al. 2008) & 7 \\
\hline Auxin-responsive proteins & Developmental processes; stress signaling (Kazan and Manners 2009) & 6 \\
\hline Chitinase proteins & Plant defense; response to plant-parasitic nematode (PPN) (Grover 2012; Sato et al. 2019) & 5 \\
\hline MYB transcription factors & Plant defense (Dubos et al. 2010) & 4 \\
\hline Pathogenesis-related proteins & Defense responses (Breen et al. 2017) & 4 \\
\hline Proteinase inhibitors & Resistance against PPN (McPherson and Harrison 2001) & 4 \\
\hline Detoxification-related proteins & Removal of toxins; reduction of oxidative stress (Blokhina et al. 2003; Gillet et al. 2017) & 4 \\
\hline $\mathrm{ABC}$ transporters & Plant growth and development; detoxification; defense (Hwang et al. 2016) & 4 \\
\hline Resistance proteins & Resistance pathways (Jones and Dangl 2006; Takken et al. 2006) & 1 \\
\hline Histone deacetylase 6 & Regulation of gene expression; responses to stress (Vijayapalani et al. 2018) & 1 \\
\hline Hypersensitive-induced reaction protein 4 & Plant defense reactions ( $\mathrm{Li}$ et al. 2019) & 1 \\
\hline Nep1-interacting protein & $\begin{array}{l}\text { Plant defense and cell death (Bailey 1995; Ottmann et al. 2009; Qutob et al. 2006; Zhang et al. } \\
\text { 2012) }\end{array}$ & 1 \\
\hline
\end{tabular}
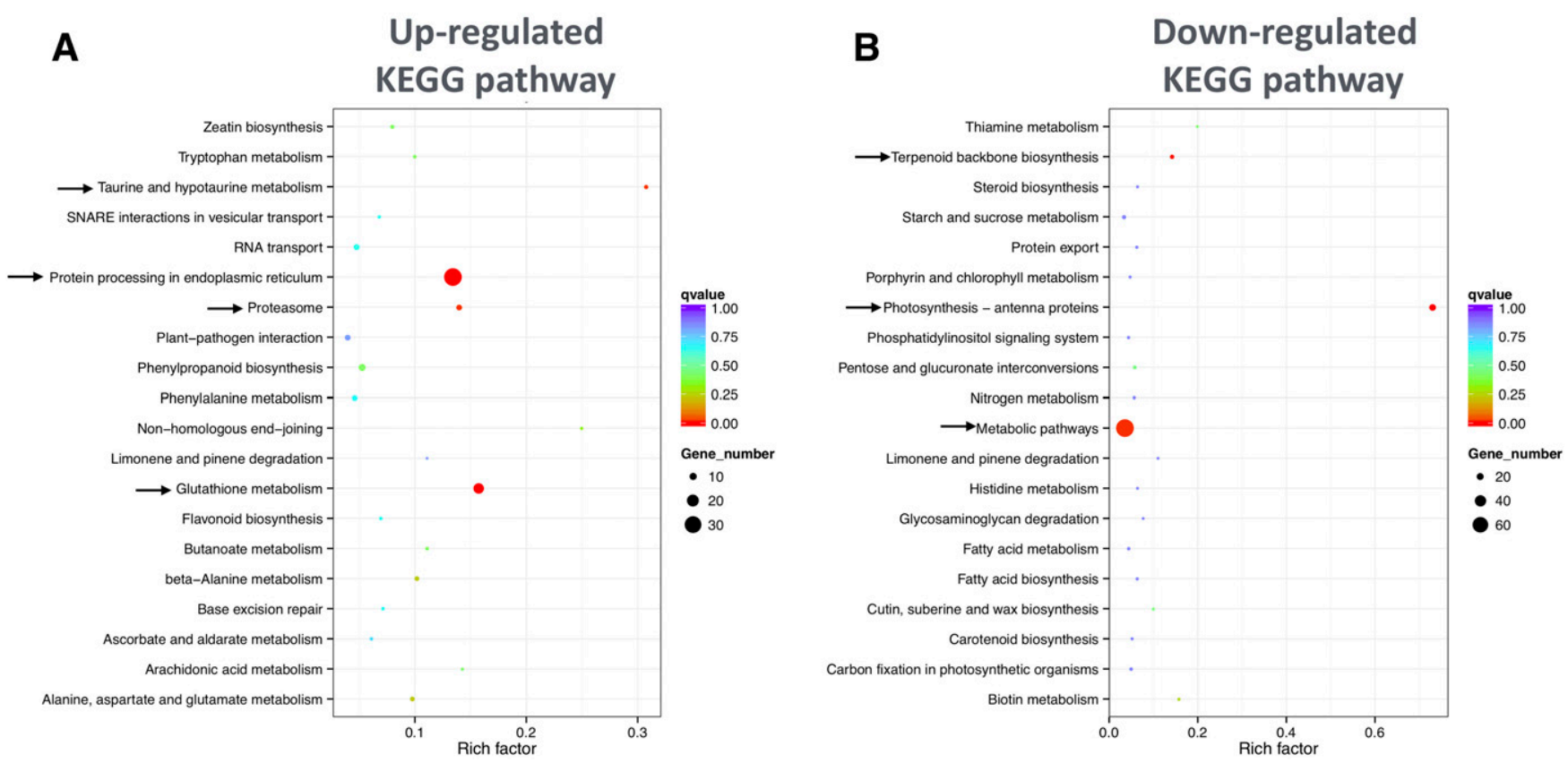

Fig. 6. Kyoto Encyclopedia of Genes and Genomes (KEGG) enrichment scatter plot analysis showing the total number of genes in A, the most represented upregulated and $\mathbf{B}$, downregulated pathways. The colored dots correspond to the $q$ value and the dot size indicates the number of differentially expressed genes mapped to the reference pathways. Arrows indicate the significantly enriched KEGG pathways $(q$ values $<0.05)$. 
potato cyst nematode effector RHA1B was shown to be a ubiquitin ligase that was able to manipulate the host ubiquitination pathway to promote parasitism by suppressing the plant immune system (Kud et al. 2019). Other reports suggest that pathogens use their effector proteins to hijack the host ubiquitin system or otherwise possess E3 activity to ubiquitinate host defense-related factors for degradation (Kud et al. 2019; Magori and Citovsky 2011; Üstün and Börnke 2014). Based on the number of highly upregulated ubiquitin-associated genes identified in this work, the Ppen 10370 mode of action during the parasitism of $P$. penetrans could potentially be related to one of these scenarios. Detection of the Ppen10370 $\Delta$ SP:eGFP fusion protein in the ER of $N$. benthamiana cells partially supports this conclusion; ubiquitin modification systems might be involved in regulation and clearance of the ER-localized proteins (Guerra and Callis 2012).

Genes downregulated during Ppen10370 expression are likely to be involved in the phenotypic characteristics of the infected plants and in the suppression of host defense reactions. Downregulation of chloroplast genes was previously recorded during overexpression of another RLN effector, an expansin-like protein Pp-EXPB1 in $N$. benthamiana plants (Vieira and Nemchinov 2020). A large-scale downregulation of photosynthesisrelated genes may also constitute an adaptive response to biotic stress by means of redirecting host resources to immediate defense needs (Bilgin et al. 2010). Downregulation of a cluster of genes encoding LRR receptor-like serine/threonine kinases, one of the central components of the plant immune system and pathogen recognition (Afzal et al. 2008), could be in line with the potential Ppen10370 activity related to ubiquitination of host defense-related factors for degradation. Defects in plant growth and appearance and venation patterning observed in PVX/Ppen10370-infected plants were likely due to the downregulation of several genes encoding auxin-related proteins. Decreased activities of cellulose synthases, integral membrane proteins whose sole function is production of the biopolymer cellulose, and polygalacturonases, pectin-modifying enzymes, indicate involvement in the processes of deconstruction of the plant cell wall.

In summary, based on the results obtained in this study and, particularly, on the gene expression analysis, we propose that function of the Ppen 13070 candidate effector protein is pertinent to the diversion of the host ubiquitin system to promote parasitism of $P$. penetrans. This function appears to be critical for the nematode. RNA silencing experiments demonstrated that knocking down this gene hampered nematode development. Therefore, although the detailed mechanisms of the Ppen 13070 action remain to be determined, our research provided an insight into its complex interaction with the host, which is likely aimed at manipulating host defense-related factors to ferry them for degradation. Validating the putative roles of the orthologs genes from other RLN could provide additional evidence on the importance of Ppen13070 for parasitism across different species of this genus. The identification of the core effectors like Ppen 13070 that are critical for RLN parasitism is essential to achieve specific control measures against this group of plant-parasitic nematodes.

\section{MATERIALS AND METHODS}

\section{Nematodes and plants.}

Pratylenchus penetrans NL 10p RH collected in Beltsville, MD, U.S.A., was maintained and multiplied in vitro in roots of corn (Zea mays L. cv. Iochief) growing in Murashige and Skoog basal medium containing vitamins and 3\% (wt/vol) sucrose $(\mathrm{pH}$ 5.8 ) that was solidified with $1.5 \%$ (wt/vol) agar (Sigma-Aldrich). Nematodes were extracted from infected corn roots as described by Vieira et al. (2015). $N$. benthamiana plants were grown in a greenhouse for 4 to 6 weeks at approximately $25^{\circ} \mathrm{C}$ with a photoperiod of $14 \mathrm{~h}$ of light and $10 \mathrm{~h}$ of dark.

\section{Amplification and cloning of nematode candidate effectors.}

Primers were designed for PCR amplification of both genomic DNA and complementary DNA (cDNA) of the predicted candidate effector sequences (Supplementary Table S5). DNA extraction was performed from mixed stages (eggs, juveniles, females, and males) of $P$. penetrans, using the PureLink genomic DNA mini kit (Invitrogen), following manufacturer instructions. Total RNA was extracted from mixed life stages of $P$. penetrans as well as $P$. neglectus and $P$. thornei, using the RNeasy plant mini kit (Qiagen), according to manufacturer instructions. RNA was then treated with RNase-Free DNase before RT. First-strand cDNA was synthesized using the iScript first-strand synthesis kit (Bio-Rad), following manufacturer instructions. Each PCR product was cloned into pCR II-TOPO vector (Thermo Fisher Scientific), followed by verification using automated Sanger sequencing in both directions (Macrogen Corp.).

\section{Sequence analysis.}

The cloned cDNA and genomic sequences were aligned using the MUSCLE tool (Edgar 2004), and gene schematics were generated with the Exon-Intron Graphic Maker (available online). The nucleotide and translated amino acid sequences were searched for homologs using BLAST analyses against the National Center for Biotechnology Information (NCBI) nonredundant nucleotide and protein databases. Sequence homology was also analyzed against publicly available nematode genomes (WormBase). Local tBLASTn searches were performed against the transcriptomes of $P$. brachyurus, $P$. coffeae (Haegeman et al. 2011), P. neglectus, P. thornei, P. zeae (FosuNyarko et al. 2016). For $P$. brachyurus (BioProject PRJNA551772), $P$. neglectus and P. thornei (BioProject PRJNA512537 [Okubara et al. 2019]) de novo assemblies were generated using the corresponding raw reads deposited at the NCBI Sequence Read Archive (SRA). Protein sequence analysis was conducted using

Table 3. Selected proteins encoded by genes downregulated in response to transient expression of the Pratylenchus penetrans Ppen10370 effector gene

\begin{tabular}{llc}
\hline Name & \multicolumn{1}{c}{ Relevant functional roles } \\
\hline Chloroplastic proteins & Photosynthesis-related; adaptation to biotic stress (Bilgin et al. 2010; Zhao et al. 2016) \\
Receptor-like serine/threonine kinase & Signal perception; pathogen recognition (Afzal et al. 2008) \\
Auxin-related proteins & Developmental processes; stress signaling (Ljung 2013) \\
bHLH transcription factors & Plant development, stress response; homeostasis, metabolism (Feller et al. 2011) \\
Cellulose synthase & Cellulose synthesis (Richmond 2000) & 66 \\
Sucrose synthase-like proteins & Disruption of sugar metabolism (Stein and Granot 2019) & 5 \\
Subtilisin-like proteases & Plant-pathogen recognition and immune priming (Figueiredo et al. 2014) \\
Polygalacturonase & Pectin-modifying enzymes (Hadfield and Bennett 1998) \\
QUIRKY proteins & Plant organ development and morphology (Fulton et al. 2009) \\
Sieve element occlusion protein & Wound sealing of the phloem (Ernst et al. 2012) \\
\hline
\end{tabular}


SignalP 4.0 for prediction of protein signal peptides (Petersen et al. 2011) and CLC Main Workbench (v.8.0) software for determination of the protein molecular mass and theoretical isoelectric points. Predicted functional domains were scanned using Pfam and InterproScan software packages (EMBL-EBI).

\section{Differential expression}

of $P$. penetrans candidate effector genes.

Transcriptome data of $P$. penetrans-infected plants were extracted from the BioProjects PRJNA304159 and PRJNA547347 of the NCBI SRA (Vieira et al. 2015, 2019). The expression of the candidate effector genes among the different transcriptome libraries was calculated as reads per kilobase of transcript per million mapped reads values. Semiquantitative RT-PCR analyses were performed using total RNA extracted from the nematode-infected roots of alfalfa cv. Baker at 3 and 7 DAI, following the same methodology as described by Vicente et al. (2019). The following genes were used as references: NP_001237047 for alfalfa (Postnikova et al. 2013) and $18 S$ rRNA gene for P. penetrans (Supplementary Table S5).

Table 4. Differential gene expression profile ( $\log _{2}$-fold change) for 25 selected genes of potato virus X (PVX)/Ppen10370 versus PVX/WT (wild type, no insert) infected leaves of Nicotiana benthamiana ${ }^{\mathrm{a}}$

\begin{tabular}{|c|c|c|c|}
\hline Gene_ID & Blast Swiss Prot & RNA-seq & qPCR \\
\hline Niben101Scf09044g01012.1 & P14170|OSMO_TOBAC Osmotin & 5.11 & 6.89 \\
\hline Niben101Scf07491g00003.1 & P08252|CHI1_TOBAC Endochitinase & 4.07 & 5.2 \\
\hline Niben101Scf01015g01002.1 & P09762|WIN2_SOLTU Wound-induced protein WIN2 & 3.56 & 4.12 \\
\hline Niben101Scf04053g02006.1 & P11670|PRB1_TOBAC Basic form of pathogenesis-related protein 1 & 3.46 & 3.99 \\
\hline Niben101Scf02349g03002.1 & P12437|PERX_SOLTU Suberization-associated anionic peroxidase & 3.07 & 4.63 \\
\hline Niben101Scf08196g01001.1 & P25317|GSTXA_TOBAC Probable glutathione S-transferase & 2.97 & 3.51 \\
\hline Niben101Scf00046g02008.1 & Q6NQK2|NAC8_ARATH NAC domain-containing protein 8 & 2.41 & 3.88 \\
\hline Niben101Scf05123g02015.1 & Q8BKD6|R144B_MOUSE E3 ubiquitin-protein ligase & 2.30 & 3.21 \\
\hline Niben101Scf05528g01009.1 & Q94F47|UBC28_ARATH Ubiquitin-conjugating enzyme & 2.26 & 4.95 \\
\hline Niben101Scf01294g05002.1 & Q6R567|RMA1_CAPAN E3 ubiquitin-protein ligase & 2.21 & 4.12 \\
\hline Niben101Scf01400g00014.1 & P13046|PRR1_TOBAC Pathogenesis-related protein R major form & 2.15 & 3.61 \\
\hline Niben101Scf01773g08011.1 & Q6R567|RMA1_CAPAN E3 ubiquitin-protein ligase & 1.95 & 4.76 \\
\hline Niben101Scf15364g00004.1 & Q9CA23|UFM1_ARATH Ubiquitin-fold modifier 1 & 1.55 & 1.09 \\
\hline Niben101Scf04801g01004.1 & Q8GYT9|SIS3_ARATH E3 ubiquitin-protein ligase & 1.42 & 7.50 \\
\hline Niben101Scf01681g07003.1 & F4JKK0|SUD1_ARATH Probable E3 ubiquitin ligase & 1.38 & 2.25 \\
\hline Niben101Scf08130g00016.1 & Q03662|GSTX1_TOBAC Probable glutathione S-transferase & 1.34 & 2.28 \\
\hline Niben101Scf12160g00012.1 & Q810L3|CHFR_MOUSE E3 ubiquitin-protein ligase & 1.15 & 2.45 \\
\hline Niben101Scf07528g00003.1 & Q6R567|RMA1_CAPAN E3 ubiquitin-protein ligase & 1.11 & 3.22 \\
\hline Niben101Scf00352g02004.1 & Q8LPN7|RNG1L_ARATH E3 ubiquitin-protein ligase RING1-like & 1.08 & 1.34 \\
\hline Niben101Scf23606g00005.1 & Q40374|PR1_MEDTR Pathogenesis-related protein PR-1 & -1.05 & -2.23 \\
\hline Niben101Scf27265g00006.1 & Q9SS87|SEOB_ARATH Protein SIEVE ELEMENT OCCLUSION B & -1.65 & -1.97 \\
\hline Niben101Scf13600g01004.1 & P27492|CB21_TOBAC Chlorophyll a-b binding protein 16, chloroplastic & -1.78 & -2.01 \\
\hline Niben101Scf00887g01011.1 & Q9FX32|SUS6_ARATH Sucrose synthase 6 & -1.94 & -2.75 \\
\hline Niben101Scf08044g00001.1 & P28493|PR5_ARATH Pathogenesis-related protein 5 & -3.12 & -4.01 \\
\hline Niben101Scf12954g02010.1 & Q948T9|RBOHB_SOLTU Respiratory burst oxidase homolog protein B & -4.80 & -4.03 \\
\hline
\end{tabular}

a As detected by RNA-sequencing (RNA-seq) data and validated by quantitative real-time PCR (qPCR).
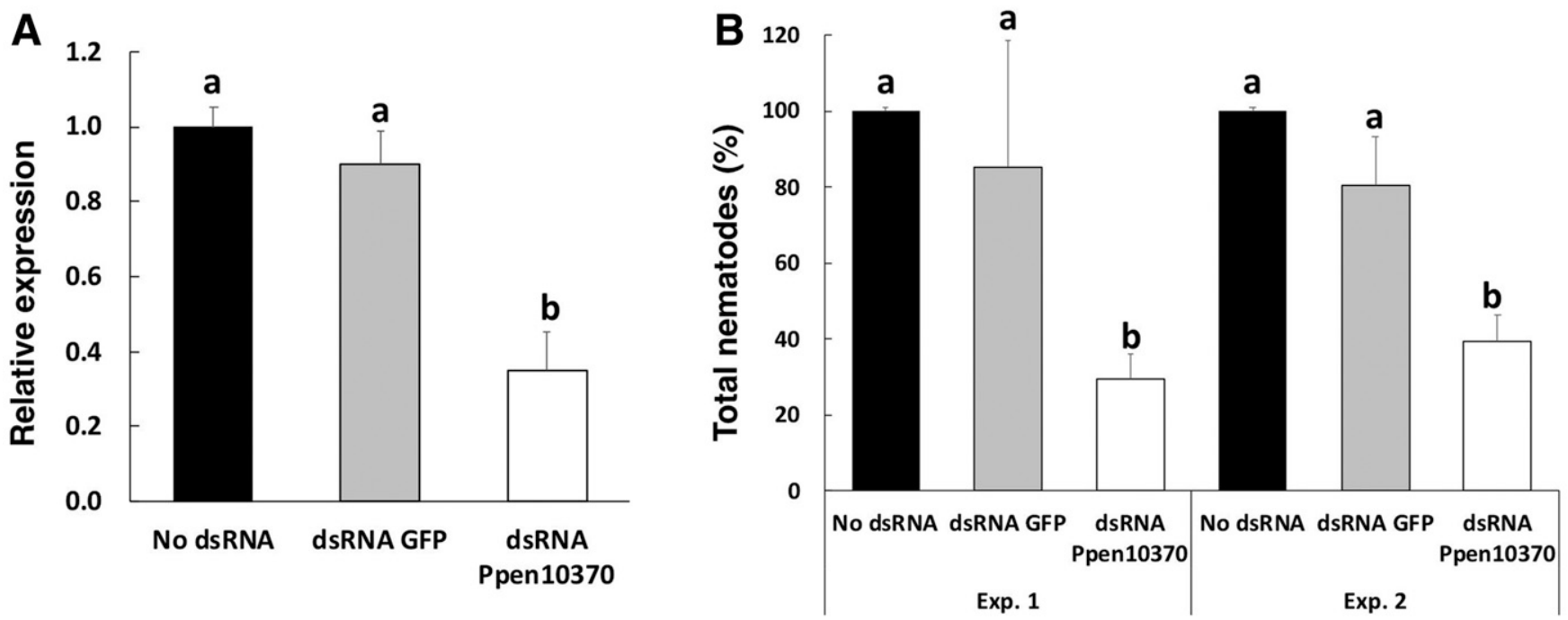

Fig. 7. Silencing of the Pratylenchus penetrans Ppen10370 gene diminished nematode development. A, Relative abundance of Ppen10370 transcripts determined by reverse transcription-quantitative PCR after nematode soaking RNA interference silencing assays. For each treatment, the gene silencing effect was evaluated $24 \mathrm{~h}$ after soaking (no double-stranded [ds]RNA corresponds to the soaking solution only; dsRNA GFP; and dsRNA Ppen10370). B, Total number of nematodes recovered from carrot disks after 6-weeks is presented as relative percentage against the treatment with soaking solution only (no dsRNA) in two independent assays. Error bars represent the standard error of the mean. 


\section{Generation of PVX-based constructs and expression in planta.}

To express each individual candidate effector in planta, EcoRV-flanked PCR products of transcripts encoding the mature protein (without signal peptide) were cloned into the pCR II-TOPO vector (Thermo Fisher Scientific) according to the manufacturer directions and were verified by automated Sanger sequencing. The corresponding plasmids were digested with the EcoRV restriction enzyme, were gel-purified, and were subcloned into the EcoRV-linearized PVX-based vector pP2C2S (obtained from D. Baulcombe, Sainsbury Laboratories, Norwich, U.K.). The integrity of all PVX clones was verified by automated sequencing. The $\mathrm{pP} 2 \mathrm{C} 2 \mathrm{~S}$ plasmids were linearized with SpeI, and capped transcripts were generated from the cDNA clones using the Ambion T7 mMessage machine kit (Thermo Fisher Scientific). Transcripts of each individual PVX construct were mechanically inoculated into fully expanded leaves of $N$. benthamiana, $N$. tabacum, and Solanum lycopersicum plants. Transcripts were also produced from pP2C2S plasmids without inserts ("empty" PVX vector) and were inoculated into plants to serve as controls representing a wild-type PVX infection (PVX/WT). Plants inoculated with buffer were used as negative ("healthy") controls. Inoculated plants were monitored daily for symptoms. The inoculation experiments included all sets of plants (a minimum of three) of each variant repeated three times. At 14 DAI, the leaves were photographed, were collected, were snap-frozen in liquid nitrogen, and were stored at $-80^{\circ} \mathrm{C}$ until RNA extraction.

\section{Western blotting.}

Western blot assays were performed, essentially as described in Nemchinov and Natilla (2007), with leaves from N. benthamiana plants infected with PVX/Ppen10370 and PVX/WT. Membranes were probed with mouse monoclonal anti-GFP antibodies (Roche Applied Science) followed by phosphataselabeled affinity purified antibody to mouse immunoglobulin $\mathrm{G}$ (Kirkegaard and Perry Laboratories, Inc.). Reactions were developed with a 1-Step NBT/BCIP phosphatase substrate (Thermo Fisher Scientific).

\section{Subcellular localization in plant cells.}

The coding sequence of Ppen10370 effector gene without signal peptide was amplified by PCR using specific primers (Supplementary Table S5) and was cloned into the Gateway donor vector $\mathrm{pCR} 8 / \mathrm{GW} / \mathrm{TOPO}$ (Thermo Fisher Scientific). The sequences were then subcloned, using Gateway LR clonase (Thermo Fisher Scientific), into the GFP fusion vector pK7FWG2.0 (Karimi et al. 2002; provided by J. T. Jones of The James Hutton Institute, Dundee, U.K.), as a C-terminal fusion (Ppen10370 $\Delta$ SP:eGFP). Competent cells of Agrobacterium tumefaciens EHA105 were transformed by the corresponding recombinant pK7FWG2.0 plasmid and were plated on Luria Broth (LB) agar containing rifampicin and kanamycin at a concentration of $50 \mu \mathrm{g} / \mathrm{ml}$, following the protocol of Hammond et al. (2019). After incubation at $28^{\circ} \mathrm{C}$, the transformed A. tumefaciens was grown in $5 \mathrm{ml}$ of liquid LB overnight at $28^{\circ} \mathrm{C}$ at $250 \mathrm{rpm}$ in a shaking incubator. The bacteria were then centrifuged for $10 \mathrm{~min}$ at $4,000 \times g$ and were resuspended in $2 \mathrm{ml}$ of the infiltration medium $(10 \mathrm{mM}$ morpholineethane sulfonic acid, $10 \mathrm{mM} \mathrm{MgCl}_{2}, \mathrm{pH} 5.7,2 \mathrm{mM}$ acetosyringone). After incubation at room temperature for $4 \mathrm{~h}$, the culture was adjusted to an optical density at $600 \mathrm{~nm}=0.05$ and was infiltrated into three to four leaves of $N$. benthamiana plants using a needleless syringe. Infiltrated leaf patches were mounted in water and images were taken with an inverted confocal microscope (Axiovert 200 M, LSM510 META; Zeiss). GFP expression was detected after excitation with a 488-nm wavelength laser and emissions collected with a 499 to $550 \mathrm{~nm}$ beam path.

\section{RNA-seq experiments.}

For Illumina RNA-seq experiments, total RNA extraction was collected using the RNeasy plant mini kit (Qiagen) following manufacturer instructions. Three biological replicates (five pooled leaves from each of the three plants represented one replicate) were used for PVX/Ppen10370-infected plants. PVX/WT-infected plants were used as control. The purity and quantity of the samples were verified with a NanoDrop spectrophotometer (ThermoFisher Scientific). RNA-seq was performed by Novogene USA. cDNA libraries were generated using a poly (A) selection method, and paired-end reads $(2 \times$ $150 \mathrm{bp}$ ) were obtained on the Illumina HiSeq 2500 platform.

\section{Read mapping and functional analysis.}

The paired-end reads were mapped onto the available genome sequence of $N$. benthamiana downloaded from the Sol Genomics Network (Bombarely et al. 2012) and to the representative genome of PVX (NCBI accession number M72416.1), using the RNA-seq analysis module in CLC Genomics Workbench (v.12) software (Qiagene). Gene annotations were based on BLASTX hits with the Swiss-Prot database. The DESeq2 package from Bioconductor in the $R$ statistics suite ( $R$ version 3.4.0) was used to estimate the sample quality and expression levels of the genes. Genes with a fold change $>2$ and a FDR $<0.05$ were counted as DEGs. Part of the computational analyses related to GO and KEGG enrichment was performed by the RNA-seq provider Novogene USA. Significant GO and KEGG enrichment terms were detected by Fisher's exact test (FDR < 0.05).

\section{Verification of transcriptome data by qPCR.}

qPCR was performed with 25 selected genes to confirm the transcriptomic data. Primers were designed using the online Realtime PCR tool (Integrated DNA Technologies Inc) (Supplementary Table S5). cDNA for qPCR analyses was synthesized using the iScript cDNA synthesis kit (Bio-Rad), following manufacturer instructions, with the same RNA samples that were used for RNA-seq. Amplification was conducted with a CFX96 real-time system machine (Bio-Rad), with three biological replicates, using the following parameters: $95^{\circ} \mathrm{C}$ for $10 \mathrm{~min}(1 \mathrm{cycle})$ followed by $95^{\circ} \mathrm{C}$ for $10 \mathrm{~s}$ and $60^{\circ} \mathrm{C}$ for $45 \mathrm{~s}$ (40 cycles). The $2^{-\Delta \Delta C t}$ method was used for the analysis of relative expression (Livak and Schmittgen 2001). The L23 and PP2A genes (Liu et al. 2012) from $N$. benthamiana were used as internal reference genes to determine relative expression values.

\section{Nematode soaking silencing assays by RNA interference.}

For the RNA interference (RNAi) silencing assay, mixed stages of $P$. penetrans were soaked in dsRNA matching $212 \mathrm{bp}$ of the coding sequence of the Ppen 10370 effector gene. As control, dsRNA matching a 715-bp fragment of GFP sequence was used. dsRNA sequences were synthesized with the Ambion MEGAscript RNAi kit (Thermo Fisher Scientific) by in vitro transcription, using Ppen1037- or GFP-specific PCR products as a template. The RNAi soaking assay followed methodologies previously applied for other Pratylenchus species (Joseph et al. 2012; Tan et al. 2013). Approximately 1,500 mixed stages (juveniles and adults) of $P$. penetrans were incubated in $200 \mu \mathrm{l}$ of RNAi soaking solution composed of M9 buffer, $50 \mathrm{mM}$ octopamine (Sigma-Aldrich), $3 \mathrm{mM}$ spermidine (SigmaAldrich), and $1 \mathrm{mg} / \mathrm{ml}(150 \mu \mathrm{l})$ of the corresponding dsRNA construct. As control, nematodes were soaked with dsRNAGFP or with the soaking solution only. Nematodes were then 
incubated for $24 \mathrm{~h}$ at $28^{\circ} \mathrm{C}$ in the dark. After incubation, an aliquot of the soaked nematodes (approximately 220 nematodes) was washed with M9 buffer solution and was used for RNA extraction as described above and, consequently, for qPCR analyses, in order to validate Ppen10370 gene silencing. To test the effect of RNAi on nematode infectivity, nematodes were then inoculated into sterilized carrot discs and were maintained for six weeks at $25^{\circ} \mathrm{C}$. A minimum of six carrot discs were used per treatment (Ppen10370-dsRNA, dsRNAGFP, and soaking solution only, respectively). After six weeks, nematodes were extracted from the carrot discs and were counted. Two independent biological experiments were performed. Statistically significant differences between treatments $(P<0.05)$ were determined by a one-way analysis of variance and pos hoc comparisons using Tukey's test.

\section{ACKNOWLEDGMENTS}

We thank I. Zasada for providing nematodes of Pratylenchus neglectus and $P$. thornei.

\section{AUTHOR-RECOMMENDED INTERNET RESOURCES}

\section{EMBL-EBI InterPro:}

https://www.ebi.ac.uk/interpro

NCBI databases: https://www.ncbi.nlm.nih.gov

Realtime PCR tool:

https://www.idtdna.com/scitools/Applications/RealTimePCR

Sol Genomics Network:

https://solgenomics.net/organism/Nicotiana_benthamiana/genome

WormBase: https://wormbase.org

WormWeb Exon-Intron Graphic Maker: WormWeb.org

\section{LITERATURE CITED}

Afzal, A. J., Wood, A. J., and Lightfoot, D. A. 2008. Plant receptor-like serine threonine kinases: Roles in signaling and plant defense. Mol. Plant-Microbe Interact. 21:507-517.

Bailey, B. A. 1995. Purification of a protein from culture filtrates of Fusarium oxysporum that induces ethylene and necrosis in leaves of Erythroxylum coca. Phytopathology 85:1250-1255.

Bartel, B., and Citovsky, V. 2012. Focus on ubiquitin in plant biology. Plant Physiol. 160:1.

Bilgin, D. D., Zavala, J. A., Zhu, J., Clough, S. J., Ort, D. R., and DeLucia, E. H. 2010. Biotic stress globally downregulates photosynthesis genes. Plant Cell Environ. 33:1597-1613.

Blokhina, O., Virolainen, E., and Fagerstedt, K. V. 2003. Antioxidants, oxidative damage and oxygen deprivation stress: A review. Ann. Bot. 91: 179-194.

Bombarely, A., Rosli, H. G., Vrebalov, J., Moffett, P., Mueller, L. A., and Martin, G. B. 2012. A draft genome sequence of Nicotiana benthamiana to enhance molecular plant-microbe biology research. Mol. PlantMicrobe Interact. 25:1523-1530.

Breen, S., Williams, S. J., Outram, M., Kobe, B., and Solomon, P. S. 2017. Emerging insights into the functions of pathogenesis-related protein 1. Trends Plant Sci. 22:871-879.

Castillo, P., and Vovlas, N. 2007. Pratylenchus (Nematoda: Pratylenchidae): diagnosis, biology, pathogenicity and management. Page 529 in: Nematology Monographs and Perspectives. D. J. Hunt, and R. N. Perry, eds. Vol. 6. Brill, Leiden, Netherlands

Chapman, S., Kavanagh, T., and Baulcombe, D. 1992. Potato virus X as a vector for gene expression in plants. Plant J. 2:549-557.

Davis, E. L., and MacGuidwin, A. E. 2000. Lesion nematode disease. The Plant Health Instructor. American Phytopathology Society, St. Paul, MN, U.S.A.

Dubos, C., Stracke, R., Grotewold, E., Weisshaar, B., Martin, C., and Lepiniec, L. 2010. MYB transcription factors in Arabidopsis. Trends Plant Sci. 15:573-581.

Edgar, R. C. 2004. MUSCLE: A multiple sequence alignment method with reduced time and space complexity. BMC Bioinformatics 5:113.

Ernst, A. M., Jekat, S. B., Zielonka, S., Müller, B., Neumann, U., Rüping, B., Twyman, R. M., Krzyzanek, V., Prüfer, D., and Noll, G. A. 2012. Sieve element occlusion (SEO) genes encode structural phloem proteins involved in wound sealing of the phloem. Proc. Natl. Acad. Sci. U.S.A. 109:E1980-E1989.

Feller, A., Machemer, K., Braun, E. L., and Grotewold, E. 2011. Evolutionary and comparative analysis of MYB and bHLH plant transcription factors. Plant J. 66:94-116.

Figueiredo, A., Monteiro, F., and Sebastiana, M. 2014. Subtilisin-like proteases in plant-pathogen recognition and immune priming: A perspective. Front. Plant Sci. 5:739.

Fosu-Nyarko, J., Tan, J. A., Gill, R., Agrez, V. G., Rao, U., and Jones, M. G. K. 2016. De novo analysis of the transcriptome of Pratylenchus zeae to identify transcripts for proteins required for structural integrity, sensation, locomotion and parasitism. Mol. Plant Pathol. 17:532-552.

Fulton, L., Batoux, M., Vaddepalli, P., Yadav, R. K., Busch, W., Andersen, S. U., Jeong, S., Lohmann, J. U., and Schneitz, K. 2009. DETORQUEO, OUIRKY, and ZERZAUST represent novel components involved in organ development mediated by the receptor-like kinase STRUBBELIG in Arabidopsis thaliana. PLoS Genet. 5:e1000355.

Gillet, F. X., Bournaud, C., Antonino de Souza Júnior, J. D., and Grosside-Sa, M. F. 2017. Plant-parasitic nematodes: Towards understanding molecular players in stress responses. Ann. Bot. 119:775-789.

Grover, A. 2012. Plant chitinases: Genetic diversity and physiological roles. Crit. Rev. Plant Sci. 31:57-73.

Guerra, D. D., and Callis, J. 2012. Ubiquitin on the move: The ubiquitin modification system plays diverse roles in the regulation of endoplasmic reticulum- and plasma membrane-localized proteins. Plant Physiol. 160: 56-64.

Gullner, G., Komives, T., Király, L., and Schröder, P. 2018. Glutathione Stransferase enzymes in plant-pathogen interactions. Front. Plant Sci. 9 1836

Hadfield, K. A., and Bennett, A. B. 1998. Polygalacturonases: Many genes in search of a function. Plant Physiol. 117:337-343.

Haegeman, A., Joseph, S., and Gheysen, G. 2011. Analysis of the transcriptome of the root lesion nematode Pratylenchus coffeae generated by 454 sequencing technology. Mol. Biochem. Parasitol. 178:7-14.

Hammond, R. W., Swift, S. M., Foster-Frey, J. A., Kovalskaya, N. Y., and Donovan, D. M. 2019. Optimized production of a biologically active Clostridium perfringens glycosyl hydrolase phage endolysin PlyCP41 in plants using virus-based systemic expression. BMC Biotechnol. 19:101.

Hwang, J.-U., Song, W. Y., Hong, D., Ko, D., Yamaoka, Y., Jang, S., Yim, S., Lee, E., Khare, D., Kim, K., Palmgren, M., Yoon, H. S., Martinoia, E., and Lee, Y. 2016. Plant ABC Transporters enable many unique aspects of a terrestrial plant's lifestyle. Mol. Plant 9:338-355.

Jones, J. D., and Dangl, J. L. 2006. The plant immune system. Nature 444: 323-329.

Joseph, S., Gheysen, G., and Subramaniam, K. 2012. RNA interference in Pratylenchus coffeae: Knock down of Pc-pat-10 and Pc-unc-87 impedes migration. Mol. Biochem. Parasitol. 186:51-59.

Karimi, M., Inzé, D., and Depicker, A. 2002. GATEWAY vectors for Agrobacterium-mediated plant transformation. Trends Plant Sci. 7 : 193-195.

Kazan, K., and Manners, J. M. 2009. Linking development to defense: Auxin in plant-pathogen interactions. Trends Plant Sci. 14:373-382.

Kud, J., Wang, W., Gross, R., Fan, Y., Huang, L., Yuan, Y., Gray, A., Duarte, A., Kuhl, J. C., Caplan, A., Goverse, A., Liu, Y., Dandurand, L. M., and Xiao, F. 2019. The potato cyst nematode effector RHA1B is a ubiquitin ligase and uses two distinct mechanisms to suppress plant immune signaling. PLoS Pathog. 15:e1007720.

Lacomme, C., and Chapman, S. 2008. Use of potato virus X (PVX)-based vectors for gene expression and virus-induced gene silencing (VIGS). Curr. Protoc. Microbiol. 8:16I.1.1-16I.1.13.

Li, S., Zhao, J., Zhai, Y., Yuan, Q., Zhang, H., Wu, X., Lu, Y., Peng, J., Sun, Z., Lin, L., Zheng, H., Chen, J., and Yan, F. 2019. The hypersensitive induced reaction 3 (HIR3) gene contributes to plant basal resistance via an EDS1 and salicylic acid-dependent pathway. Plant J. 98:783-797.

Liu, D., Shi, L., Han, C., Yu, J., Li, D., and Zhang, Y. 2012. Validation of reference genes for gene expression studies in virus-infected Nicotiana benthamiana using quantitative real-time PCR. PLoS One 7:e46451.

Livak, K. J., and Schmittgen, T. D. 2001. Analysis of relative gene expression data using real-time quantitative PCR and the $2^{-\Delta \Delta C t}$ method. Methods 25:402-408.

Ljung, K. 2013. Auxin metabolism and homeostasis during plant development. Development 140:943-950.

Magori, S., and Citovsky, V. 2011. Hijacking of the host SCF ubiquitin ligase machinery by plant pathogens. Front. Plant Sci. 2:87.

Marino, D., Peeters, N., and Rivas, S. 2012. Ubiquitination during plant immune signaling. Plant Physiol. 160:15-27. 
McPherson, M. J., and Harrison, D. J. 2001. Protease inhibitors and directed evolution: Enhancing plant resistance to nematodes. Biochem. Soc. Symp. 68:125-142.

Nemchinov, L. G., and Natilla, A. 2007. Transient expression of the ectodomain of matrix protein 2 (M2e) of avian influenza A virus in plants. Protein Expr. Purif. 56:153-159.

Nomura, H., Komori, T., Uemura, S., Kanda, Y., Shimotani, K., Nakai, K., Furuichi, T., Takebayashi, K., Sugimoto, T., Sano, S., Suwastika, I. N., Fukusaki, E., Yoshioka, H., Nakahira, Y., and Shiina, T. 2012. Chloroplast-mediated activation of plant immune signalling in Arabidopsis. Nat. Commun. 3:926.

Nuruzzaman, M., Sharoni, A. M., and Kikuchi, S. 2013. Roles of NAC transcription factors in the regulation of biotic and abiotic stress responses in plants. Front. Microbiol. 4:248.

Okubara, P. A., Peetz, A. B., and Sharpe, R. M. 2019. Cereal root interactions with soilborne pathogens-From trait to gene and back. Agronomy (Basel) 9:188.

Ottmann, C., Luberacki, B., Küfner, I., Koch, W., Brunner, F., Weyand, M., Mattinen, L., Pirhonen, M., Anderluh, G., Seitz, H. U., Nürnberger, T., and Oecking, C. 2009. A common toxin fold mediates microbial attack and plant defense. Proc. Natl. Acad. Sci. U.S.A. 106: 10359-10364.

Pandey, S. P., and Somssich, I. E. 2009. The role of WRKY transcription factors in plant immunity. Plant Physiol. 150:1648-1655.

Park, C. J., and Seo, Y. S. 2015. Heat shock proteins: A review of the molecular chaperones for plant immunity. Plant Pathol. J. 31:323-333.

Petersen, T. N., Brunak, S., von Heijne, G., and Nielsen, H. 2011. SignalP 4.0: Discriminating signal peptides from transmembrane regions. Nat. Methods 8:785-786.

Pichersky, E., and Raguso, R. A. 2018. Why do plants produce so many terpenoid compounds? New Phytol. 220:692-702.

Postnikova, O. A., Shao, J., and Nemchinov, L. G. 2013. Analysis of the alfalfa root transcriptome in response to salinity stress. Plant Cell Physiol. 54:1041-1055.

Qutob, D., Kemmerling, B., Brunner, F., Küfner, I., Engelhardt, S., Gust, A. A., Luberacki, B., Seitz, H. U., Stahl, D., Rauhut, T., Glawischnig, E., Schween, G., Lacombe, B., Watanabe, N., Lam, E., Schlichting, R., Scheel, D., Nau, K., Dodt, G., Hubert, D., Gijzen, M., and Nürnberger, T. 2006. Phytotoxicity and innate immune responses induced by Nep1-like proteins. Plant Cell 18:3721-3744.

Richmond, T. 2000. Higher plant cellulose synthases. Genome Biol. 1: 3001.1-3001.6.

Sato, K., Kadota, Y., and Shirasu, K. 2019. Plant immune responses to parasitic nematodes. Front. Plant Sci. 10:1165.

Serrano, I., Audran, C., and Rivas, S. 2016. Chloroplasts at work during plant innate immunity. J. Exp. Bot. 67:3845-3854.

Smant, G., Stokkermans, J. P. W. G., Yan, Y., de Boer, J. M., Baum, T. J., Wang, X., Hussey, R. S., Gommers, F. J., Henrissat, B., Davis, E. L., Helder, J., Schots, A., and Bakker, J. 1998. Endogenous cellulases in animals: Isolation of $\beta$-1, 4-endoglucanase genes from two species of plant-parasitic cyst nematodes. Proc. Natl. Acad. Sci. U.S.A. 95: 4906-4911.

Stein, O., and Granot, D. 2019. An overview of sucrose synthases in plants. Front. Plant Sci. 10:95.

Takken, F. L. W., Albrecht, M., and Tameling, W. I. L. 2006. Resistance proteins Molecular switches of plant defence. Curr. Opin. Plant Biol. 9:383-390.

Tan, J. A. C. H., Jones, M. G. K., and Fosu-Nyarko, J. 2013. Gene silencing in root lesion nematodes (Pratylenchus spp.) significantly reduces reproduction in a plant host. Exp. Parasitol. 133:166-178.

Üstün, S., and Börnke, F. 2014. Interactions of Xanthomonas type-III effector proteins with the plant ubiquitin and ubiquitin-like pathways. Front. Plant Sci. 5:736.

Varshavsky, A. 2008. Discovery of cellular regulation by protein degradation. J. Biol. Chem. 283:34469-34489.

Vicente, C. S. L., Nemchinov, L. G., Mota, M., Eisenback, J. D., Kamo, K., and Vieira, P. 2019. Identification and characterization of the first pectin methylesterase gene discovered in the root lesion nematode Pratylenchus penetrans. PLoS One 14:e0212540.

Vieira, P., Eves-van den Akker, S., Verma, R., Wantoch, S., Eisenback, J. D., and Kamo, K. 2015. The Pratylenchus penetrans transcriptome as a source for the development of alternative control strategies: Mining for putative genes involved in parasitism and evaluation of in planta RNAi. PLoS One 10:e0144674.

Vieira, P., and Gleason, C. 2019. Plant-parasitic nematode effectors-Insights into their diversity and new tools for their identification. Curr. Opin. Plant Biol. 50:37-43.

Vieira, P., Maier, T. R., Eves-van den Akker, S., Howe, D. K., Zasada, I., Baum, T. J., Eisenback, J. D., and Kamo, K. 2018. Identification of candidate effector genes of Pratylenchus penetrans. Mol. Plant Pathol. 19:1887-1907.

Vieira, P., Mowery, J., Eisenback, J. D., Shao, J., and Nemchinov, L. G. 2019. Cellular and transcriptional responses of resistant and susceptible cultivars of alfalfa to the root lesion nematode, Pratylenchus penetrans. Front. Plant Sci. 10:971.

Vieira, P., and Nemchinov, L. G. 2020. An expansin-like candidate effector protein from Pratylenchus penetrans modulates immune responses in Nicotiana benthamiana. Phytopathology 110:684-693.

Vijayapalani, P., Hewezi, T., Pontvianne, F., and Baum, T. J. 2018. An effector from the cyst nematode Heterodera schachtii derepresses host rRNA genes by altering histone acetylation. Plant Cell 30:2795-2812.

Zhang, H., Li, D., Wang, M., Liu, J., Teng, W., Cheng, B., Huang, Q., Wang, M., Song, W., Dong, S., Zheng, X., and Zhang, Z. 2012. The Nicotiana benthamiana mitogen-activated protein kinase cascade and WRKY transcription factor participate in Nep1(Mo)-triggered plant responses. Mol. Plant-Microbe Interact. 25:1639-1653.

Zhao, J., Zhang, X., Hong, Y., and Liu, Y. 2016. Chloroplast in Plant-Virus Interaction. Front. Microbiol. 7:1565.

Zunke, U. 1990. Observations on the invasion and endoparasitic behavior of the root lesion nematode Pratylenchus penetrans. J. Nematol. 22: 309-320. 